\title{
The enlarged spleen - a blood bank
}

\section{Rajneesh Kumar Singh}

Dept of Surgical Gastroenterology

Sanjay Gandhi Post Graduate

Institute of Medical Sciences

(SGPGIMS)

Lucknow

India

\section{Correspondence:}

Dr Rajneesh Kumar Singh

Email: rajneeshkumarsingh@ hotmail.com
Splenomegaly may be the result of several diseases such as myeloproliferative disorders, portal hypertension etc. Sometimes the splenic size may assume massive proportions. A massive splenomegaly may lead to symptoms or changes in laboratory parameters over and above the original disease state. The predominant symptoms include pain due to the splenic infarcts, dragging sensation due to the sheer size, early satiety etc. It has been known for several decades that a large spleen is a massive pool of sequestered blood cells. ${ }^{1}$ It can be holding $30-90 \%$ of all platelets and $40 \%$ of all erythrocytes. ${ }^{2}$ The bone marrow is typically hypercellular to compensate for this sequestration.

Splenectomy entails removal of the whole spleen along with the sequestered blood cells in the spleen. This seems like a terrible waste of the sequestered blood components. If one was able to harvest these components the potential benefits could include a rise in the hematocrit, possibly avoidance of blood transfusion, immediate rise in platelet count, with implications for hemostasis and a rise in leucocyte count. The potential secondary benefits may include easier handling of a smaller spleen, reduced blood loss and lesser operation time.

While it is quite common practice to ligate the splenic artery first, during splenectomy for a massive splenomegaly, the objective benefits of this step have been studied by few. This step of the surgical procedure is commonly performed by surgeons to reduce the problems of handling a massive spleen and, possibly, reduce blood loss during peri-splenic dissection, particularly in patients with portal hypertension. The 'auto-transfusion' of sequestered components is but a side-effect of this step. One concern of such an auto-transfusion is the quality of blood cells being released into the blood stream. Other potential concerns are the risks of dissecting the large tortuous splenic artery, especially in patients with portal hypertension, in the face of unquantified benefits.

The study by Madhavan et al in this issue of the journal studied the objective benefit of early ligation of the splenic artery during splenectomy for portal hypertension. ${ }^{3}$ They studied 30 patients with portal hypertension (NCPF 20, EHPVO 9, Cirrhosis 1) who underwent splenectomy and proximal spleno-renal shunt in their unit. They found that there is significant increase in the RBC and platelet counts, though there was no statistically significant increase in the total leucocyte count, differential leucocyte count and hemoglobin. Even though the study suffers from several methodologic flaws it does bring into focus the auto-transfusion benefits of early ligation of the splenic artery during splenectomy. However the study does not report the percentage changes in the cell counts and only the mean values have been used for analysis instead of paired analysis.

Gazula et al had earlier studied a similar population and reported a significant increase in the hemoglobin, hematocrit, leucocyte, platelet and RBC counts after early ligation of the splenic artery. ${ }^{4}$ They had restricted intravenous fluid administration after splenic artery ligation and thus found that benefits extended to increased hemoglobin, hematocrit and leucocyte count also. Both the studies excluded patients requiring intravenous fluids or blood transfusion and did not include a control population. While this may have removed the confounding 
effect of intravenous fluids on the study data, but the addition of a control arm and effect on blood loss etc, would have increased the clinical applicability of these studies.

According to Madhavan et al the net gain from ligation of splenic artery to 30 minutes after splenectomy for RBC was $1.00+0.23 \mathrm{mill} / \mathrm{cumm}$ (mean $+\mathrm{SD}$ ), and for platelet was 1,09,500.0 +46765.9 cells/cumm (mean $+\mathrm{SD})$. The platelet increase was equivalent to transfusion of 8 units of platelets. Similar findings had been seen by Gazula et al. This group had found a median rise in hemoglobin of $0.45 \mathrm{~g} / \mathrm{dl}$, a median rise in hematocrit of $1.2 \%$, median rise in TLC of $2500 / \mathrm{mm}^{3}$ and a median rise in platelets of $19500 / \mathrm{mm}^{3}$, after early ligation of splenic artery. This, according to them, was equivalent of transfusion of half unit of packed RBC and 4 units of platelets.

These two studies also demonstrated that the blood cells sequestered in the spleen are otherwise normal. Madhavan et al reported that the $\mathrm{MCV}, \mathrm{MCH}$ and $\mathrm{MCHC}$ are unchanged before or after splenectomy. Hence the fears of releasing deformed cells into the circulation seem to be unfounded.

It is probably oversimplification to suggest that hypersplenism is an invariable accompaniment of splenic enlargement. Studies on association of splenic volume with hematological parameters have not shown consistent results. While a study on Gaucher's disease patients showed a negative correlation, another large study suggested that patients with liver disease had better correlation than patients with hematologic disorders. ${ }^{5,6}$ Even the splenic sequestration seems to affect some components more than others. While thrombocytopenia is quite common with large splenomegaly, leucopenia is not that common. The classical hematologic picture of hypersplenism is further complicated by the underlying disease that may also be contributing to the low counts. For example low blood cell counts in patients with cirrhosis may also be due to impaired production, anti-viral therapy or increased destruction.

The diagnosis of hypersplenism seems fundamental to the idea of auto-transfusion during splenectomy. However studies on auto-transfusion during splenectomy have not dealt with this point with clarity. ${ }^{3,4}$ Though the post-splenectomy hematologic picture may show dramatic changes in patients with preoperative hypersplenism, but some increase in counts may occur even in patients without overt preoperative hypersplenism.

Thus it would be desirable to know which patients would benefit most from splenic auto-transfusion. The epinephrine stimulation test provides just this promise. Simply put, subcutaneously injected epinephrine leads to splenic contraction and rise in the blood cell counts. Therefore hypersplenism and auto-transfusion could be predicted even without splenectomy. Misselwitz et al demonstrated safety and feasibility of the test and proposed cutoff values to improve specificity of the test. ${ }^{7}$ However it has lacked wider applicability and needs to be tested in more centers.

The concept of splenic auto-transfusion may be complemented by manual compression of the spleen after splenic artery ligation. This was practiced by Madhavan et al in their study. ${ }^{3}$ The potential benefit of manual compression would be difficult to quantify and the amount of applied pressure difficult to objectivize. Further, a potential adverse event such as splenic rupture is always a possibility. It has been demonstrated that epinephrine causes splenic contraction in animal experiments and a few human case series. The knowledge that epinephrine causes splenic contraction can be used to improve splenic auto-transfusion. ${ }^{7-9}$ Currently, a study evaluating the safety and feasibility of splenic artery injection of epinephrine during splenectomy is being conducted at my Institution. Preliminary results indicate that it is a safe procedure and improves the auto-transfusion during splenectomy.

Auto-transfusion during splenectomy is application of knowledge gained several decades ago from physiology research. However some aspects have remained unresolved, such as predictive factors for increase in the blood counts, implications of auto-transfusion in myeloproliferativedisorders, etc. Further studies should include nuclear medicine studies to measure splenic volume and a control arm to study the true clinical benefit of these modifications of splenectomy.

\section{References}

1. Aster RH. Pooling of platelets in the spleen: role in the pathogenesis of "hypersplenic" thrombocytopenia. J Clin Invest. 1966;45:645-57.

2. Jandl JH, Aster RH. Increased splenic pooling and the pathogenesis of hypersplenism. Am J Med Sci. 1967;253:383-98.

3. Madhavan M, Vimalraj V, Selvakumar E, et al. Hematologic changes following early ligation of splenic artery during splenectomy in shunt surgery for portal hypertension. Trop Gastroenterol. 2012;33:51-4.

4. Gazula S, Pawar DK, Seth T, Bal CS, Bhatnagar V. Extrahepatic portal venous obstruction: The effects of early ligation of splenic artery during splenectomy. I Indian Assoc Pediatr Surg. 2009;14:194-9. 
5. Gielchinsky Y, Elstein D, Hadas-Halpern I, Lahad A, Abrahamov A, Zimran A. Is there a correlation between degree of splenomegaly, symptoms and hypersplenism? A study of 218 patients with Gaucher disease. Br J Haematol. 1999;106:812-6.

6. Misselwitz B, Bachli EB, Kaiser P, Fehr J, Goede JS. Diagnosis of hypersplenism with the epinephrine stimulation test - 23 years of experience at a tertiary care hospital. Swiss Med Wkly. 2012;141:w13324.

7. Pastorini C, Iannetti M, Martini U, Masperone MA, Ronzitti $\mathrm{M}$, Rosselli P, et al. Effect of adrenaline on the splanchnic venous blood flow in splenectomized dogs and splenomegalic dogs. Minerva Gastroenterol. 1970;16:154-8.

8. Branehög I, Weinfeld A, Roos B. The exchangeable splenic platelet pool studied with epinephrine infusion in idiopathic thrombocytopenic purpura and in patients with splenomegaly. Br J Haematol. 1973;25:239-48.

9. Espersen K, Frandsen H, Lorentzen T, Kanstrup IL, Christensen NJ. The human spleen as an erythrocyte reservoir in divingrelated interventions. J Appl Physiol. 2002;92:2071-9. 